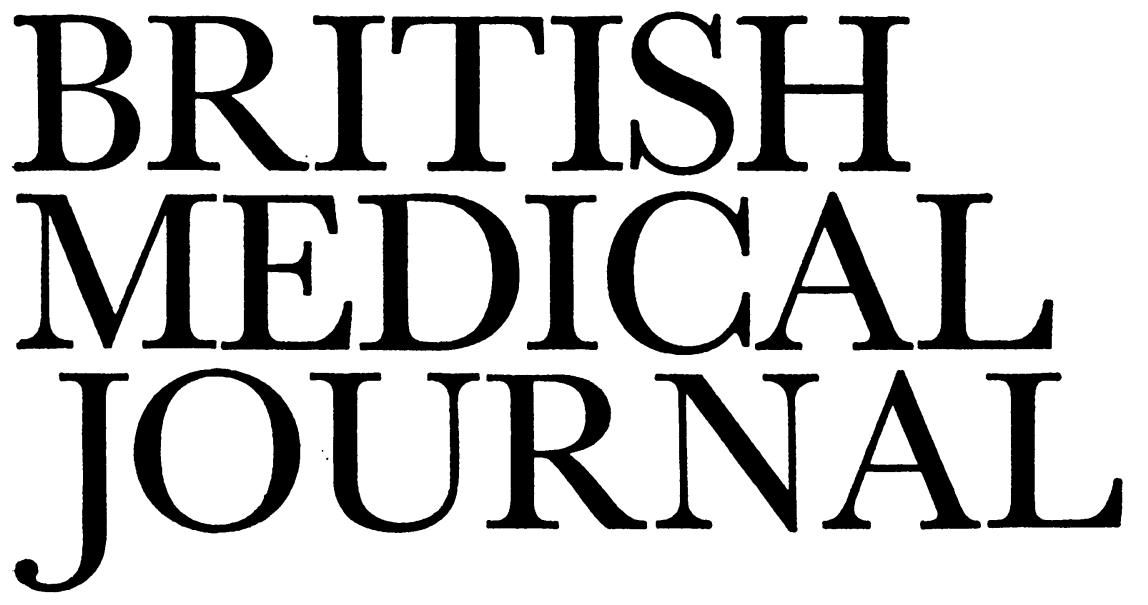

LONDON, SATURDAY 16 JULY 1977

\title{
What causes toxicity in uraemia?
}

Many and various are the substances that accumulate in the body fluids of patients with uraemia. ${ }^{1}$ Simple substances such as water, $\mathrm{H}^{+}, \mathrm{Na}^{+}, \mathrm{K}^{+}$, and phosphate may all be toxic when retained in the body in excessive amounts; but the symptoms of uraemia may occur even when water and acid base balance, as well as mineral metabolism, are not grossly abnormal. The explanation usually advanced is that other substances must accumulate in uraemia and act as uraemic toxins, though in only a few instances have single compounds been shown to cause specific symptoms or complications.

For example, parathyroid hormone is present in excessive amounts in uraemia and is (in part at least) responsible for uraemic osteodystrophy. The excess of parathyroid hormone is not, however, due to limited renal excretion but to increased secretion secondary to changes in calcium, phosphate, and vitamin D metabolism-an example of Bricker's "trade-off" hypothesis. ${ }^{2}$ This argues that certain hormones which may exert toxic effects accumulate in uraemia not because of reduced renal excretion but from a homoeostatic adaptation to a reduced glomerular filtration rate. Bricker's group ${ }^{34}$ has also shown that a natriuretic factor or hormone stimulated by volume expansion accumulates in severe renal failure, and they have suggested that toxic levels of this substance inhibit active transport of sodium and other substances in body cells. A fraction with a molecular weight between 500 and 1000 daltons inhibits sodium transport in vitro and in vivo.

Many other substances accumulate in patients with uraemia. Among those suspected of being uraemic toxins have been urea, creatinine, uric acid, methylguanidine, guanidinosuccinic acid, other guanidines, amino-acids, amines both aliphatic and aromatic, phenols, indoles, aromatic oxyacids, pseudouridine, oxalic acid, magnesium, arsenic, myoinositol, "middle molecules," glucagon, and growth hormone.

The success of haemodialysis using conventional cellophane or cuprophane membranes in correcting many uraemic symptoms suggested that the uraemic toxins must have molecular weights of less than 5000 , since these membranes are impermeable to larger molecules. Substances with small molecular weights such as urea, creatinine, and uric acid can cross these membranes readily, and it was thought at first that uraemic toxins were of this general size. In 1971, however, Babb et $a l^{5}$ introduced the square metre hour hypothesis, which postulated that retention of "middle molecules" with a molecular weight between 500 and 5000 played an important part in the toxic manifestations of uraemia, particularly the peripheral neuropathy. This hypothesis was based on two clinical observations. Firstly, patients on regular peritoneal dialysis developed peripheral neuropathy less often (despite higher blood urea and creatinine concentrations) than patients on regular haemodialysis-presumably because the peritoneum was more permeable to middle molecules than cellophane; and, secondly, the prevention of peripheral neuropathy in patients on regular haemodialysis seemed to depend more on the number of hours of dialysis per week than on the maintenance of specific predialysis concentrations of urea and creatinine. Removal of middle molecules during haemodialysis was, according to $\mathrm{Babb}$ et $a l,{ }^{6}$ dependent more on dialyser surface area and the duration of dialysis than on blood and dialysate flow rates, though later the relationship was found to be rather more complex than had first been supposed.

No middle molecules have been isolated or proved to cause specific symptoms or complications. Even so, these hypotheses have had a considerable effect on the design of new dialysers and on new dialysis protocols. There has been a vogue for using large surface area dialysers or new membranes more permeable to the supposed middle molecules. Some groups have reduced the time on dialysis by using dialysers with large surface areas. Other workers, however, have claimed good results from shortening dialysis times while still using conventional dialysers. ${ }^{7}$ Numerous studies, some taking theoretical clearance of middle molecules into account and others not, have yielded confusing and conflicting results. ${ }^{8} 9$ Von Hartitzsch et al ${ }^{10}$ pointed out that in many instances the effect of residual renal function had not been considered and that inaccurate determinations of middle molecule clearances by dialysers may also have confused the picture. Certainly other factors seem more important in initiating neuropathy: severe clinical uraemia before the start of regular dialysis treatment predisposes to peripheral neuropathy, and it is more likely to progress in uncooperative patients with uncontrolled hypertension, with hyperkalaemia, and with excessive fluid weight gains between dialyses.

Recently several groups have shown that solutes in the 
middle molecule range accumulate in the blood of uraemic patients. Bergström et al, ${ }^{11}$ using chromatographic techniques, found eight or nine distinct peaks. Peak No 7, corresponding to a molecular weight in the range of 1000 to 2000 daltons, was in the plasma of severely uraemic patients but not in normal plasma; it could be further separated into subfractions. Peaks high in uraemic plasma were also excreted in increased amounts in the urine, suggesting that accumulations of these middle molecule fractions occur as a result of increased production and not reduced renal excretion. There was a clinical impression that patients with complications such as pericarditis or peripheral neuropathy had higher individual peaks than those without.

Direct evidence of toxicity of middle molecule fractions is limited. An inhibitory effect on glucose utilisation has been reported by Dzurik et al. ${ }^{12}$ Man et al ${ }^{13}$ reported that a middle molecule fraction inhibited fibroblast proliferation; Odeberg et $a l^{14}$ found an inhibitory effect on granulocyte function, and Bergström ${ }^{11}$ reported one on lymphoblastic transformation in vitro.

In addition to the possible role of middle molecules as uraemic toxins there is also evidence that some uraemic toxins may have molecular weights below 500. A cytotoxic effect in vitro has been reported with a small molecular weight fraction (containing guanidine derivatives) derived from uraemic sera by Yamada and Nakagawa. ${ }^{15} \mathrm{Ku}$ et al ${ }^{16}$ reported inhibition by methylguanidine and guanidine of lymphoblastic transformation in vitro.

There is little evidence that creatinine itself is toxic, but enteric cycling of creatinine does occur, and several metabolites have been identified including $\mathrm{N}$-methylhydantoin, creatine, sarcosine, glyoxylate, and methylamine-some of which may be toxic. ${ }^{1718}$ Of the various nitrogenous substances which are retained in uraemia, urea itself seems to be the least toxic, although Johnson et al $^{19}$ found some evidence that urea may contribute to nausea, vomiting, lethargy, headaches, and the haemorrhagic tendency.

So what we know about uraemic toxicity is still confusing. The uraemic syndrome seems to be due to many uraemic toxins, some with low molecular weights and others in the so-called middle molecule range. Clearly much more work must be done in isolating and identifying uraemic toxins and also in determining their biological effects. The possible relevance of depletion of essential substances by dialysis itself must also be explored.

${ }^{1}$ Bergström, J, Proceedings of the European Dialysis and Transplant Association, 1975, 12, 579.

2 Bricker, N S, New Ensland fournal of Medicine, 1972, 286, 1093.

3 Bricker, N S, Bourgoignie, J J, and Klahr, S, Archives of Internal Medicine, 1970, 126, 860.

${ }^{4}$ Bourgoignie, J J, et al, fournal of Clinical Investigation, 1972, 51, 1514.

5 Babb, A L, et al, Transactions. American Society for Artificial Internal Organs, 1971, XVII, 81.

6 Babb, A L, et al, Transactions. American Society for Artificial Internal Organs, 1972, XVIII, 98.

${ }^{7}$ Cambi, V, et al, Proceedings of the European Dialysis and Transplant Association, 1973 10, 271.

${ }^{8}$ Kjellstrand, C M, et al, Transactions. American Society for Artificial Internal Organs, 1973, XIX, 325.

${ }^{9}$ Man, N K, et al, Proceedings of the European Dialysis and Transplant Association, 1973, 10, 236.

10 Von Hartitzsch, B, et al, Proceedings of the European Dialysis and Transplant Association, 1973, 10, 522.

11 Bergström, J, et al, in Proceedings of the 6th International Congress of Nephrology, p 600, ed S Giovannetti, V, Bonomini, and G D'Amico, Basel, Karger, 1976.

12 Dzurik, R, et al, Clinica Chimica Acta, 1973, 46, 77

${ }_{13}$ Man, N K, et al, Proceedings of the European Dialysis and Transplant Association, 1974, 11, 214.

14 Odeberg, H, Olsson, I, and Thysell, H, Transactions. American Society for Artificial Internal Organs, 1973, XIX, 484.
15 Yamada, T, and Nakagawa, S, Transactions. American Society for Artificial Internal Organs, 1976, XXII, 155.

${ }^{16} \mathrm{Ku}, \mathrm{G}$, et al, Proceedings of the European Dialysis and Transplant Association, $1974,11,427$.

17 Jones, J D, and Burnett, P C, Kidney International, 1975, 7, Suppl 3, 294.

$1 *$ Simenhoff, M L, Kidney International, 1975, 7, suppl 3, 314.

${ }^{19}$ Johnson, W J, et al, Kidney International, 1975, 7, suppl 3, 288.

\section{Diagnostic laparotomy}

The perplexed and puzzled physician may ask a surgical colleague to perform a diagnostic laparotomy to help solve either of two comparatively common clinical problems: pyrexia of undetermined origin (PUO) and undiagnosed abdominal pain. What are the chances of success?

Reviewing recent reports, Rothman et al have found that a positive diagnosis was made at laparotomy for PUO in $57-82^{\circ}{ }_{0}$ of cases. In their own series of 24 patients, all of whom had undergone very extensive investigations, including even percutaneous kidney and liver biopsies, the findings were positive in no fewer than $21\left(87^{\circ}{ }_{0}\right)$. The most common diagnosis was an inflammatory process (13 patients), including hepatic granuloma, hepatitis, liver abscess, tuberculous peritonitis, and renal inflammatory disease. Neoplasia accounted for the fever in seven patients (including three with disseminated carcinomatosis), while the final patient was found to have a primary volvulus of the omentum with associated omental infarction. In three patients the diagnostic laparotomy failed to identify any lesion, but two of these patients lost their fever after operation.

Such results certainly justify laparotomy when less invasive methods of investigating a prolonged fever (which today would include computerised axial tomography and ultrasound) fail to identify its source. The place of diagnostic laparotomy in a patient with chronic abdominal pain of unknown origin, however, is less clear cut. Devor and Knauft ${ }^{2}$ gave a gloomy account of 40 such patients submitted to laparotomy, in none of whom were relevant findings made at operation. More than half had had previous surgery; three-quarters had complex emotional problems; and, not surprisingly, only 14 became asymptomatic after postoperative reassurance. In contrast, Scott et $a l^{3}$ found that laparotomy produced a positive diagnosis in 69 of 81 patients with undiagnosed "medical" disease, and Griffin ${ }^{4}$ reported positive findings at laparotomy in 21 of 24 patients with chronic abdominal pain. Eleven were found to have malignant disease and seven had inflammatory disease of the pancreas.

In the study by Rothman et al 27 patients with abdominal pain persisting for between three weeks and one year and undiagnosed in spite of intensive investigation had diagnostic laparotomies: these yielded positive findings in 22 cases $(82 \%)$, a malignant lesion being found in 13 (most often cancer of the pancreas, which was present in eight patients). Chronic pancreatitis was diagnosed in four, all of whom had a serum amylase concentration within normal limits. One had a mucocele of the appendix and one a deeply placed abdominal wall abscess. One patient had a foreign body in the submucosa of the pylorus, one had retroperitoneal fibrosis, and one had infarction of the greater omentum. All five of the patients in whom laparotomy failed to yield a diagnosis were young or middle-aged women with pain that had lasted over nine months without appreciable change in location or intensity; two lost their symptoms after surgery. 